\title{
Acute sterile endophthalmitis following intravitreal bevacizumab: case series
}

\author{
This article was published in the following Dove Press journal: \\ Clinical Ophthalmology \\ 9 September 2014 \\ Number of times this article has been viewed
}

\section{Axel Orozco-Hernández' Ximena Ortega-Larrocea' Gustavo Sánchez-Bermúdez' Gerardo García-Aguirre' Virgilio Morales Cantón' Raul Velez-Montoya ${ }^{2}$}

'Retina Department, Asociación para Evitar la Ceguera en México IAP, Mexico City, Mexico; ${ }^{2}$ Department of Ophthalmology, University of Colorado School of Medicine, Rocky Mountain Lions Eye Institute, Aurora, CO, USA
Correspondence: Raul Velez-Montoya Department of Ophthalmology, University of Colorado School of Medicine, Rocky Mountain Lions Eye Institute, 1675 Aurora Court, Aurora, CO, 80045, USA

Tel + I 7208482500

Fax +I 7208485014

Email raul.velez-montoya@ucdenver.edu
Background: Since the ophthalmological community adopted the use of intravitreal bevacizumab as an accepted off-label treatment for neovascular diseases, the amount of knowledge regarding its effects and properties has been increasing continually. In the last few years, there have been an increasing number of reports about sterile intraocular inflammation and intraocular pressure elevations after intravitreal bevacizumab. In the following case series, we describe the clinical presentation and outcomes of ten consecutive cases of patients developing mild-to-severe sterile intraocular inflammation after intravitreal bevacizumab and their management.

Methods: This report presents a retrospective case series. We reviewed the medical records of ten consecutive patients from a group of 46 , in whom repackaged bevacizumab in individual aliquots from two vials from the same batch were used. All surgical procedures were performed using standard sterile techniques in the operating room. At each follow-up visit, patients underwent a complete ophthalmological examination including visual acuity assessment, intraocular pressure, biomicroscopy, and posterior fundus examination.

Results: Ten patients presented sterile endophthalmitis with an onset time of $3.5 \pm 1.95$ days. The clinical characteristics were mild pain, slight visual loss, conjunctival hyperemia, and various degrees of intraocular inflammation with microhypopyon. All cultures were negative. All patients were managed with topical steroids and antibiotics, except two, in whom, due to severe vitreous cells, intravitreal antibiotics were used. Three patients showed a transient elevation of intraocular pressure. Only $50 \%$ of the patients regained a visual acuity equal or better to the baseline visual acuity on file.

Conclusion: The increasing number of intravitreal injections of bevacizumab applied every day, due to its widespread acceptance, might be one reason why the number of cases of sterile endophthalmitis is rising. Fast recognition and accurate differential diagnosis is important to avoid unnecessary treatments and long-term complications. The low incidence of this event should not preclude the use of intravitreal injections in eyes that could benefit greatly from this therapy.

Keywords: complications, vitrectomy, intravitreal antibiotics, pseudoendophthalmitis, bevacizumab

\section{Background}

Among the minor ophthalmological procedures, intravitreal injection of bevacizumab is probably one of the most widespread procedures. ${ }^{1-3}$ Despite its lack of approval by the US Food and Drug Administration (FDA) for ophthalmological use, ${ }^{4}$ data from the Comparison of Age-related macular degeneration Treatments Trials (CATT) demonstrated that monthly and as-needed regimes of bevacizumab are equally as effective for controlling the exudative forms of age-related macular degeneration (AMD) than the current FDAapproved drug (ranibizumab).,3,5-7 This has somehow validated bevacizumab use and reinforced a common practice in the ophthalmological community since 2005, when 
Rosenfeld et al published the first pilot study using intravitreal doses of the drug, following a small uncontrolled, open label trial that used systemic doses of bevacizumab..$^{8-11}$

Bevacizumab (Avastin ${ }^{\circledR}$; Genentech, Inc., South San Francisco, CA, USA), a full-length humanized monoclonal antibody against vascular endothelial growth factor-A (VEGF-A), has shown, repeatedly and consistently, its potential efficacy in controlling proliferative diseases and vascular leakages. ${ }^{1-3}$ Nevertheless, its intravitreal use is not entirely free of adverse effects, most of which are more related to the administration procedure than to the drug per se. ${ }^{12}$ Reports of sustained elevation of intraocular pressure and inflammation after the intravitreal use of bevacizumab have been increasing. . $^{43-17}$ Although the exact cause is yet to be determined, it is believed that the presence of particulate matter (silicon droplets or protein aggregates) due to a mishandling of the drug during storage or repackaging, as well as allergic reactions to the drug or a contaminant, might be involved. . $^{13,14,18}$

In the present series, we will describe the clinical characteristics and outcomes of ten consecutive cases of sterile endophthalmitis (defined as the acute intraocular inflammation of the vitreous cavity after an intravitreal drug that either resolves without the need of intravitreal antibiotics and/or negative culture proven), in which the administered drug came from two different vials from the same batch.

\section{Methods}

This report presents a retrospective case series. The study was approved by the internal review board of the Asociación para Evitar la Ceguera en México hospital, Mexico City, Mexico. The study was conducted according to the tenets of the Declaration of Helsinki. All patients signed informed consent forms, in which the use of an off-label drug was explained, before any procedures were done.

Using strict sterile techniques, commercially available bevacizumab (100 mg/4 mL) was divided into individual aliquots of $1.25 \mathrm{mg} / 0.05 \mathrm{~mL}$ and stored for no more than 1 week at $4^{\circ} \mathrm{C}$. All intravitreal injections were carried out in an area of the operating room specially designated for intravitreal injections only. All personnel present at the moment of injection wore surgical scrubs and face masks. Before the procedure, the attending physician washed their hands and wore sterile gloves, and an assistant cleaned the skin around the eye with a solution of povidone-iodine $10 \%$ and instilled a single drop of povidone-iodine 5\% (Betadine $^{\circledR}$; Alcon Laboratories, Inc., Fort Worth, TX, USA) in the cul-de-sac. A sterile lid speculum was used in every case. Topical proparacaine $0.5 \%$ drops were used for local anesthesia of the eye surface. All injections were done using a 27-gauge needle at 3.5-4 $\mathrm{mm}$ from the limbus (pars plana). A cotton swab was used for immediate local pressure of the injection site for a few seconds. ${ }^{12,19,20}$ Topical antibiotic prophylaxis with ciprofloxacin hydrochloride $0.3 \%$ (Ciloxan ${ }^{\circledR}$; Bausch \& Lomb Incorporated, Bridgewater, NJ, USA) was indicated in all cases for 5 days.

At each post-injection visit, all patients had a complete ophthalmological examination, which included: best corrected visual acuity (BCVA) examination; slit-lamp biomicroscopy; posterior fundus examination with indirect ophthalmoscope; intraocular pressure assessment; and screening for local and systemic adverse effects. Vitreous and aqueous samples were obtained following a strict standard protocol from all patients with suspected endophthalmitis. All samples were cultured in triplicate and checked daily for bacterial growth.

\section{Results}

We reviewed the cases of ten consecutive patients from a group of 46 patients who had intravitreal bevacizumab for a wide array of different pathologies, including choroidal neovascularization (CNV) secondary to AMD, myopic CNV, diabetic macular edema, and macular edema secondary to vein occlusion. All patients were part of the intravitreal injection clinic of the hospital and were injected on the same day in November 2012.

The first two cases of mild anterior chamber inflammation occurred within days of the injection (mean 3.5 \pm 1.95 days); thereafter, all 46 patients were seen systematically, regardless of whether or not they had symptoms. The demographic and clinical presenting characteristics of the study group patients with suspected sterile endophthalmitis are summarized in Tables 1 and 2 . The median age of the study group was $60.4 \pm 18.96$ years. All ten patients studied had variable degrees of conjunctival hyperemia, anterior chamber cells, and vitreous cells; two of these presented hypopyon and severe vitreous cells. The rest showed mild-to-moderate intraocular inflammation. All patients reported mild-to-moderate ocular pain and slight decrease of baseline visual acuity. Half of the group reported a final BCVA better or equal to the baseline BCVA on file, while the other half reported a slightly worse final BCVA (Figure 1). The visual acuity of patient number 6 was not available for analysis since he was being seen as a foreign patient and was lost to follow-up.

Although the majority of the patients did not need intraocular antibiotics or surgery for the resolution of the inflammatory process and all cultures were negative, the 
Table I Summary of demographic data at baseline

\begin{tabular}{|c|c|c|c|c|c|c|c|}
\hline Case number & Age (years) & Study eye & Diagnosis & $\begin{array}{l}\text { Number of previous } \\
\text { intravitreal injections }\end{array}$ & $\begin{array}{l}\text { BCVA } \\
\text { pretreatment }\end{array}$ & $\begin{array}{l}\text { BCVA } \\
\text { at onset }\end{array}$ & $\begin{array}{l}\text { BCVA } \\
\text { final }\end{array}$ \\
\hline 1 & 63 & OS & AMD & 4 & CF I. 5 feet & CF I.5 feet & $20 / 300$ \\
\hline 2 & 42 & OD & AMD & 0 & $20 / 50$ & $20 / 80$ & $20 / 50$ \\
\hline 3 & 71 & OD & AMD & 6 & $20 / 100$ & $20 / 800$ & $20 / 200$ \\
\hline 4 & 36 & OS & CRVO & I & CF 8 feet & CF I. 5 feet & CF I.5 feet \\
\hline 5 & 61 & OS & DME & I & CF 8 feet & $20 / 800$ & $20 / 250$ \\
\hline 6 & 36 & OD & BRVO & 0 & & & \\
\hline 7 & 59 & OD & CRVO & I & $20 / 80$ & $20 / 350$ & $20 / 150$ \\
\hline 8 & 87 & OS & CRVO & 0 & $20 / 400$ & CF 3 feet & CF 6 feet \\
\hline 9 & 90 & OS & AMD & 3 & CF I feet & CF 3 feet & CF 6 feet \\
\hline 10 & 59 & OS & BRVO & 0 & $20 / 80$ & $\mathrm{HM}$ & $20 / 200$ \\
\hline
\end{tabular}

Abbreviations: AMD, age-related macular degeneration; BCVA, best corrected visual acuity; BRVO, branch retinal vein occlusion; CF, counting fingers; CRVO, central retinal vein occlusion; DME, diabetic macular edema; HM, hand movements; OD, oculus dextrus (right eye); OS, oculus sinister (left eye).

two patients who presented with hypopyon received intravitreal antibiotic (moxifloxacin) and steroid (dexamethasone) treatment at the moment of presentation due to our inability to rule out true endophthalmitis with the clinical presentation. Nevertheless, the cultures from vitreous samples (obtained before the intravitreal antibiotics administration) were negative in both patients. Reports from pathology indicated that one of the cultures from additional samples of aqueous humor of one of the patients showed positive Staphylococcus aureus growth; however, the result was considered as a possible contamination rather than a true infectious source of inflammation, given the lack of positive growth in other control samples taken.

The rest of the patients were managed with topical antibiotics (Ocuflox ${ }^{\circledR}$ [ofloxacin 0.3\%]; Allergan Inc., Irvine, CA, USA), steroids (Prednefrin ${ }^{\circledR}$ [predonisolone 0.12\%]; Allergan Inc.), and cycloplegics (Mydryacil [tropicamide]; Alcon Laboratories, Inc.). Furthermore, three of the patients presented a transitory rise of intraocular pressure, which was adequately controlled with a short course of topical glaucoma medication. One of the patients also had severe vitreous hemorrhage $(3+)$, which resolved without treatment.

The inflammatory reaction yielded within the first week after diagnosis and vitreous cells cleared completely during the course of the first month.

Sixty percent of the patients had at least one injection of intravitreal bevacizumab before in one or both eyes. Of this group, $40 \%$ had at least one injection in the study eye before, and the rest in the contralateral eye. Regarding the baseline pathology, four patients had CNV secondary to AMD and three patients were diagnosed with macular edema secondary to central retinal vein occlusion. Two more were diagnosed with ischemic retinal vascularization secondary to branch retinal vein occlusion and one had recurrent diabetic macular edema.

\section{Representative case}

A 63-year-old Caucasian female, whose previous medical history was unremarkable for the present illness, was seen

Table 2 Summary of the clinical manifestations and complaints of the patients at onset of inflammation

\begin{tabular}{|c|c|c|c|c|c|c|c|}
\hline $\begin{array}{l}\text { Case } \\
\text { number }\end{array}$ & $\begin{array}{l}\text { Onset after } \\
\text { injection (days) }\end{array}$ & Pain & $\begin{array}{l}\text { Conjunctival } \\
\text { hyperemia }\end{array}$ & Microhypopyon & $\begin{array}{l}\text { Anterior } \\
\text { chamber cells }\end{array}$ & $\begin{array}{l}\text { Vitreous } \\
\text { cells }\end{array}$ & $\begin{array}{l}\text { Increased } \\
\text { IOP }\end{array}$ \\
\hline $\mathrm{I}$ & 3 & + & + & + & $3+$ & $3+$ & + \\
\hline 2 & 6 & + & + & + & $2+$ & + & + \\
\hline 3 & I & - & - & - & $3+$ & $\mathrm{VH}$ & - \\
\hline 4 & 7 & + & + & - & + & + & - \\
\hline 5 & 2 & + & + & - & $2+$ & $2+$ & - \\
\hline 6 & 4 & + & + & - & + & + & - \\
\hline 7 & 3 & + & + & - & $3+$ & $2+$ & - \\
\hline 8 & 4 & + & + & + & $3+$ & $2+$ & - \\
\hline 9 & 4 & + & + & + & $3+$ & $2+$ & - \\
\hline 10 & I & + & + & + & $3+$ & $3+$ & + \\
\hline
\end{tabular}

Abbreviations: IOP, intraocular pressure; $\mathrm{VH}$, vitreous hemorrhage. 


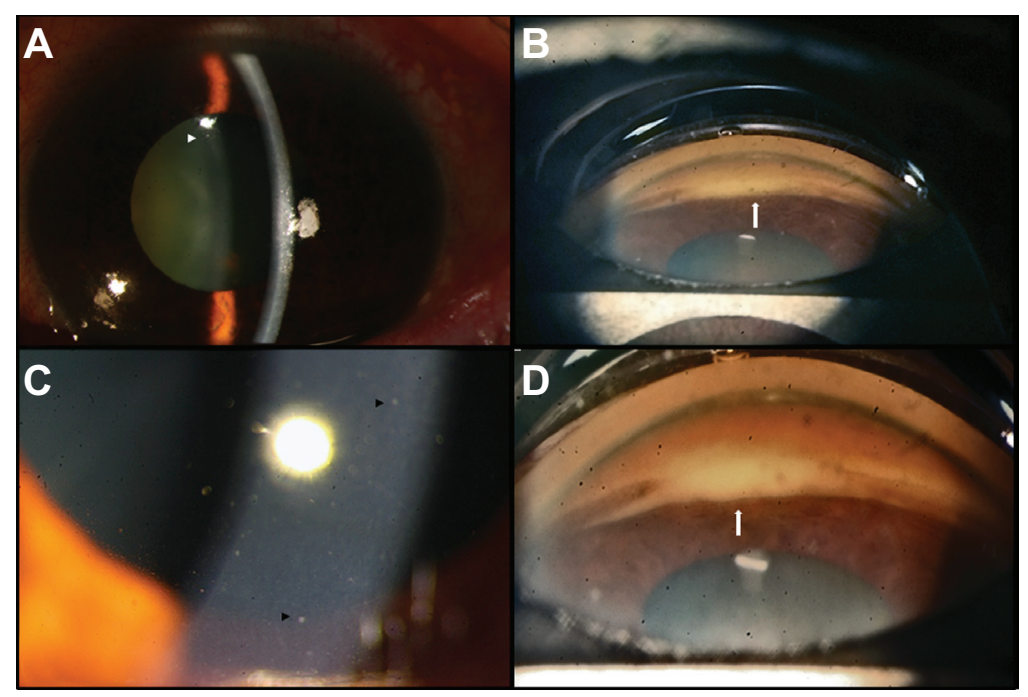

Figure I Clinical pictures.

Notes: (A) Patient number 9, mild conjunctival hyperemia. The small white arrowhead is highlighting the presence of anterior chamber cells. (B and D) Patients numbers 8 and 10, gonioscopy pictures, inferior aspect. The small white arrows show the presence of microhypopyon. (C) Patient number 2, biomicroscopic I0× image. The small black arrowheads show the presence of small keratic precipitates.

during a follow-up visit at the retina clinic due to an active CNV secondary to AMD in the left eye. The patient had been treated with monthly injections of intravitreal bevacizumab in the study eye on four previous occasions. During the follow-up consultation, the patient complained of persistent poor visual acuity in both eyes. The BCVA was 20/50 (right eye) and count fingers at 1 foot (left eye). Intraocular pressure was $12 \mathrm{mmHg}$. Anterior segment biomicroscopy of both eyes was unremarkable. Fundus examination of the left eye presented a persistent subfoveal choroidal neovascularization, with hard drusen and intraretinal cystic fluid. Fluorescein angiography showed active leakage, and spectral domain optical coherence tomography corroborated the diagnosis. The patient underwent a fifth dose of intravitreal bevacizumab using the technique and doses described previously. Three days later, the patient was seen at the emergency room complaining of visual loss, mild pain, and conjunctival hyperemia (2+). The biomicroscopy

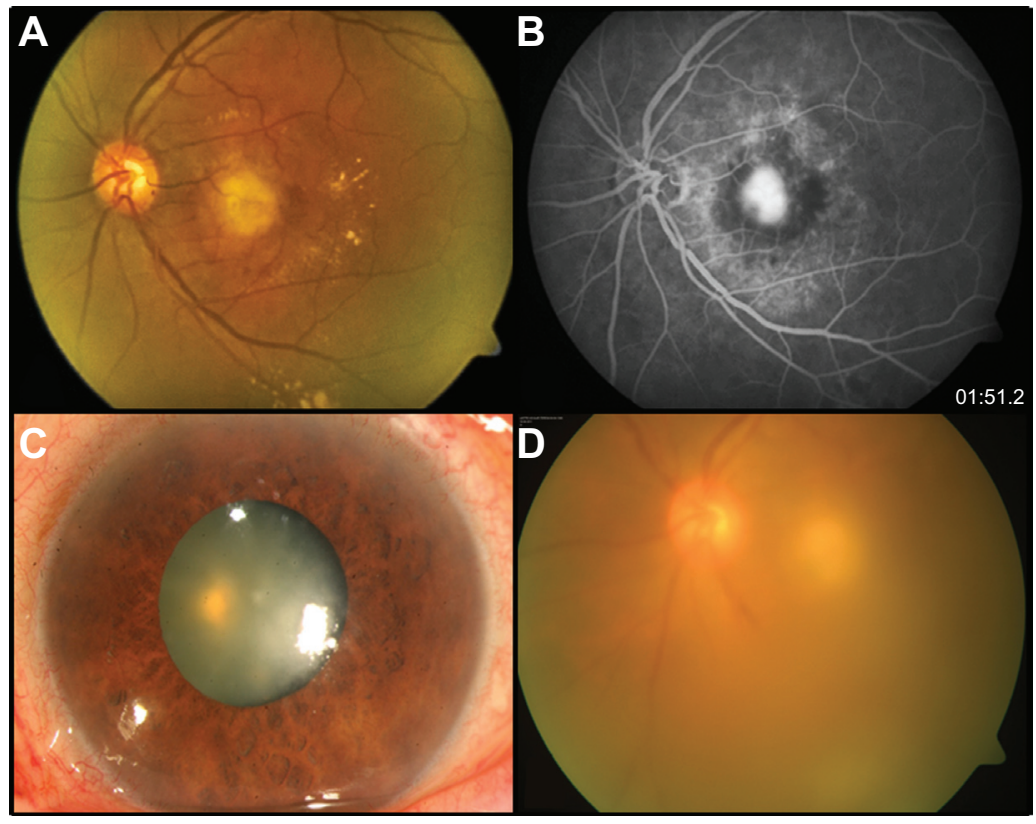

Figure 2 Patient number I, representative case.

Notes: Fundus photograph (A) and fluorescein angiography $(\mathbf{B})$ of the left eye show an active choroidal neovascularization with intraretinal fluid and leakage of dye in the fluorescein angiography. Three days later, the patient complained of mild pain, visual loss, and conjunctival hyperemia (C). Fundus photograph shows the presence of important vitreous cells (D). 
revealed fibrin in the anterior chamber, cells in aqueous and vitreous (3+) and microhypopyon. Intraocular pressure was $30 \mathrm{mmHg}$ (Figure 2). Both anterior chamber and vitreous tap were negative. The patient was treated with topical steroids, antibiotics, timolol $0.5 \%$, and dorzolamide. One week later, the inflammation had yielded, the vision had improved to $20 / 300$, and the intraocular pressure had decreased to $16 \mathrm{mmHg}$.

\section{Conclusion}

The intravitreal administration of drugs has become the preferred route for the treatment of a wide array of ocular conditions. Since the original description of the technique by Rycroft in 1945, ${ }^{21}$ the recognition of the vitreous cavity as an excellent reservoir, where relatively high levels of drugs can be maintained for a long period (typically higher concentrations than obtained by other administration routes) without incurring undesirable reactions, has changed the approach and expanded the types of drugs that can be used in the treatment of such diseases. ${ }^{18,21}$

VEGF is a glycoprotein that is secreted in the eye during ischemic conditions and which also has an important role in vascular permeability, tumor angiogenesis, and as an inflammatory molecule. ${ }^{2,3,22}$ Bevacizumab is a $149 \mathrm{kDa}$ humanized full-length monoclonal IgG antibody against VEGF with a half-life of 9.8 days in human vitreous. ${ }^{1-3,16}$ The proven noninferiority of intravitreal bevacizumab relative to its FDA-approved counterpart (ranibizumab) set a precedent for its off-label use. ${ }^{5,6}$ The safety of intravitreal injection depends not only on the surgical technique, but also on the characteristics of the administered drug; infectious endophthalmitis is a rare but potentially blinding condition that requires prompt treatment with intravitreal antibiotics and surgery. ${ }^{23}$ Certain intravitreal drugs can produce an acute and sterile intraocular inflammation that can mimic infectious endophthalmitis., ${ }^{4,24,25}$ An accurate differential between these two entities is crucial, as a delay in treatment of the former or unnecessary treatment of the latter can have major consequences. ${ }^{4}$ Another important differential that needs to be ruled out is the possibility of toxic anterior segment syndrome. ${ }^{26}$ In this case, the distinction is easier, because patients with toxic anterior segment syndrome typically develop severe anterior chamber inflammation with hypopyon just 12 to 48 hours after anterior segment procedures, conversely to sterile endophthalmitis, in which the mild symptomatology and insidious onset develops 2 days to a few weeks after injection. ${ }^{26,27}$

In the past few years, there has been an increasing number of reports of high intraocular pressure and inflammation after intravitreal use of bevacizumab. ${ }^{13,28}$ The present case series describes the clinical presentation and outcomes of ten consecutive cases that occurred after intravitreal administration of bevacizumab from two different vials from the same batch. Unfortunately, the specific batch and number were lost due to a clerical error. It is important to note that a total of 46 patients received drug from the same vials, at the same time, in the same place, and with the same technique. But only ten out of 46 patients showed manifestations of idiopathic inflammation and only three out of these ten had high intraocular pressure. In addition, one patient developed severe vitreous hemorrhage that resolved spontaneously. Although traumatic causes during injection were ruled out, the exact etiology of the hemorrhage is still uncertain and might be the result of the base pathology.

The evolution and outcome in general was similar to the outcomes described in other case series, in which, after a mild episode of inflammation, the patients recovered vision without intravitreal antibiotics or surgery. ${ }^{12,25}$ Our series seems to reinforce the notion that, although there is no clear relationship with age, sex, pathology, or number of previous intravitreal injections; sterile endophthalmitis tends to occur in clusters to occur in clusters. Interestingly, and conversely to the majority of case series published so far, our group showed slightly worse visual outcome than usual. ${ }^{17,18,25,29}$ The high variability among the baseline visual acuities prevents us from drawing any definite conclusion regarding this observation; however, the small number of cases and a worse initial visual acuity than in previously reported series may be accountable for this observation. Furthermore, although not mentioned previously, most of our patients had uncontrolled or poorly controlled concomitant systemic conditions, such as diabetes mellitus and high blood pressure, which led to poor response to treatment. This feature of our population might suggest that specific clinical conditions could be responsible for the development of sterile endophthalmitis and its evolution and treatment response, independently of the batch, contamination of aliquots, or injection technique. In our series, sterile endophthalmitis did not have any relation with previous history of surgery or inflammatory eye conditions as in other reports in the literature. ${ }^{4,12,14,17}$

The etiology of sterile endophthalmitis is poorly understood. Risk factors that seem to be related are history of uveitis, pseudophakia, and past history of vitrectomy;,29-31 however, no definite association has been proven, and there are many possible explanations. For instance, the common practice of dividing individual aliquots commercially available vials of bevacizumab, as a cost-effective method, ${ }^{23}$ may carry an increased risk of contamination with bacterial 
endotoxins. ${ }^{18,29}$ This situation may explain clusters of sterile endophthalmitis in patients treated with injections from the same batch. Furthermore, bevacizumab normal formulation may contain traces of endotoxins at levels that are deemed safe for intravenous use, but which are still capable of inducing an inflammatory reaction intravitreally. ${ }^{18,29,32}$ Another important factor is the possible existence of noninfectious particles in bevacizumab preparations. ${ }^{29}$ Evidence published by Liu et al $^{13}$ and replicated by other groups ${ }^{31,33}$ has shown that physical mishandling and extreme freeze/thaw cycles may increase the levels of silicon oil microdroplets in the syringes containing repackaged bevacizumab. ${ }^{13}$ These particles are thought to be possibly responsible for increases in intraocular pressure seen after drug administration. Temperature fluctuations have also been proposed as a factor that may increase immunogenic properties of bevacizumab. ${ }^{18}$ Temperature oscillation outside the range recommended by the manufacturer $\left(2^{\circ} \mathrm{C}-8^{\circ} \mathrm{C}\right)$ may promote protein degradation and induce novel antigenic epitopes not found in the original molecule, creating a specific immune reaction against bevacizumab. ${ }^{18}$ In addition, the relatively large molecule and extended half-life in the vitreous cavity theoretically increase this possibility. ${ }^{17}$ Finally, it is possible that compensatory elevation of angiogenic and inflammatory factors, following VEGF inhibition, may occur, promoting the clinical manifestations of sterile endophthalmitis. ${ }^{17,26,29}$

The relatively low risk of infectious endophthalmitis reported in the literature and small power of the studies to detect important adverse effects (range $0.01 \%-1.16 \%$, mean $0.0149 \% \approx 1$ in every 1,949 injections; CATT: bevacizumab $0.014 \%$, ranibizumab: $0.07 \%$ ) has precluded the elaboration of general guidelines aimed at preventing and decreasing the incidence. 5,6,23,31,34 Regarding sterile endophthalmitis, the exact incidence is unknown. The CATT reported an incidence of pseudoendophthalmitis of one out of 599 patients $(0.16 \%)$ with ranibizumab and none in a bevacizumab group. ${ }^{5,6}$ Smaller underpowered studies have reported incidences ranging from $0.09 \%$ to $1.1 \%{ }^{5,6}$ According to the United States Pharmacopeia, General Chapter 797, about pharmaceutical compounding of sterile preparations, the repackaging of multiple doses of bevacizumab is a mediumrisk compounded sterile preparation, meaning that all personnel involved in aliquot preparation must wear sterile scrubs, work under a laminar airflow workbench, and do routine disinfection and air quality testing to maintain an International Organization for Standardization (ISO) class 5 (3,520 particles $/ \mathrm{m}^{3}$ ) environment. ${ }^{23}$ In the same way, several studies, recommendations, and guidelines have been published regarding the best injection techniques in order to minimize risk of complications after intravitreal administration of the drug. ${ }^{19,20,35,36}$ Nevertheless, despite all these recommendations, cases continue to be reported worldwide.

In summary, sterile endophthalmitis and transient elevation of intraocular pressure can occur after uneventful intravitreal injections of bevacizumab, regardless of the injection technique or aliquot preparation procedure. Although there are several theories about the etiology of the intraocular inflammation, its causes are still unknown. In this case series, the majority of clinical characteristics were similar to those reported previously: presentation 2 days to 1 week after injection; mild pain, with a slight decrease in visual acuity and various degrees of intraocular inflammation; negative culture; and recovery without intravitreal antibiotics or surgery. However, conversely to previous published data, $50 \%$ of our group reported final BCVA that was worse than the baseline BCVA on file. The widespread acceptance of intravitreal bevacizumab as an accepted standard of care might explain why we are seeing these episodes more often. The general ophthalmologist as well as the retina specialist must be familiar with the clinical manifestations of these episodes in order to make good differentials, instigate proper treatment, and avoid long-term complications. Nevertheless, the extremely low incidence of such events should not prevent the intravitreal use of bevacizumab in eyes that might benefit greatly from this type of therapy.

\section{Acknowledgment}

This report was fully sponsored and conducted by the Asociación para Evitar la Ceguera Research Fund in México City.

\section{Disclosure}

The authors report no conflicts of interest in this work.

\section{References}

1. Kimoto K, Kubota T. Anti-VEGF agents for ocular angiogenesis and vascular permeability. J Ophthalmol. 2012;2012:852183.

2. Velez-Montoya R, Oliver SC, Olson JL, Fine SL, Mandava N, Quiroz-Mercado H. Current knowledge and trends in age-related macular degeneration: today's and future treatments. Retina. 2013;33(8): 1487-1502.

3. Velez-Montoya R, Oliver SC, Olson JL, Fine SL, Quiroz-Mercado H, Mandava N. Current knowledge and trends in age-related macular degeneration: genetics, epidemiology, and prevention. Retina. 2014;34(3): 423-441.

4. Bakri SJ, Larson TA, Edwards AO. Intraocular inflammation following intravitreal injection of bevacizumab. Graefes Arch Clin Exp Ophthalmol. 2008;246(5):779-781.

5. CATT Research Group, Martin DF, Maguire MG, et al. Ranibizumab and bevacizumab for neovascular age-related macular degeneration. N Engl J Med. 2011;364(20):1897-1908. 
6. Comparison of Age-related Macular Degeneration Treatments Trials (CATT) Research Group, Martin DF, Maguire MG, et al. Ranibizumab and bevacizumab for treatment of neovascular agerelated macular degeneration: two-year results. Ophthalmology. 2012;119(7):1388-1398.

7. IVAN Study Investigators, Chakravarthy U, Harding SP, et al. Ranibizumab versus bevacizumab to treat neovascular age-related macular degeneration: one-year findings from the IVAN randomized trial. Ophthalmology. 2012;119(7):1399-1411.

8. Michels S, Rosenfeld PJ, Puliafito CA, Marcus EN, Venkatraman AS Systemic bevacizumab (Avastin) therapy for neovascular age-related macular degeneration twelve-week results of an uncontrolled open-label clinical study. Ophthalmology. 2005;112(6):1035-1047.

9. Rosenfeld PJ, Moshfeghi AA, Puliafito CA. Optical coherence tomography findings after an intravitreal injection of bevacizumab (avastin) for neovascular age-related macular degeneration. Ophthalmic Surg Lasers Imaging. 2005;36(4):331-335.

10. Ziemssen F, Sobolewska B. Therapeutic efficacy of bevacizumab for age-related macular degeneration: what are the implications of CATT for routine management? Drugs Aging. 2011;28(11):853-865.

11. Jansen RM. The off-label use of medication: the latest on the Avastin - Lucentis debacle. Med Law. 2013;32(1):65-77.

12. Yamashiro K, Tsujikawa A, Miyamoto K, et al. Sterile endophthalmitis after intravitreal injection of bevacizumab obtained from a single batch Retina. 2010;30(3):485-490.

13. Liu L, Ammar DA, Ross LA, Mandava N, Kahook MY, Carpenter JF. Silicone oil microdroplets and protein aggregates in repackaged bevacizumab and ranibizumab: effects of long-term storage and product mishandling. Invest Ophthalmol Vis Sci. 2011;52(2):1023-1034.

14. Fielden M, Nelson B, Kherani A. Acute intraocular inflammation following intravitreal injection of bevacizumab - a large cluster of cases. Acta Ophthalmol. 2011;89(8):e664-e665.

15. Good TJ, Kimura AE, Mandava N, Kahook MY. Sustained elevation of intraocular pressure after intravitreal injections of anti-VEGF agents Br J Ophthalmol. 2011;95(8):1111-1114.

16. Yeh S, Albini TA, Moshfeghi AA, Nussenblatt RB. Uveitis, the Comparison of Age-Related Macular Degeneration Treatments Trials (CATT), and intravitreal biologics for ocular inflammation. Am J Ophthalmol. 2012;154(3):429-435.e2.

17. Damasceno N, Horowitz S, Damasceno E. Anterior uveitis after treatment of age-related macular degeneration with ranibizumab and bevacizumab: uncommon complication. Clin Ophthalmol. 2012;6: 1201-1205.

18. Marticorena J, Romano V, Gómez-Ulla F. Sterile endophthalmitis after intravitreal injections. Mediators Inflamm. 2012;2012:928123.

19. Green-Simms AE, Ekdawi NS, Bakri SJ. Survey of intravitreal injection techniques among retinal specialists in the United States. Am J Ophthalmol. 2011;151(2):329-332.

20. Peyman GA, Lad EM, Moshfeghi DM. Intravitreal injection of therapeutic agents. Retina. 2009;29(7):875-912.
21. Rycroft BW. Penicillin and the control of deep intra-ocular infection. Br J Ophthalmol. 1945;29(2):57-87.

22. Keane PA, Sadda SR. Development of anti-VEGF therapies for intraocular use: a guide for clinicians. J Ophthalmol. 2012;2012:483034.

23. Ng DS, Kwok AK, Chan CW, Li WW. Intravitreal bevacizumab: safety of multiple doses from a single vial for consecutive patients. Hong Kong Med J. 2012;18(6):488-495.

24. [No authors listed]. Results of the Endophthalmitis Vitrectomy Study. A randomized trial of immediate vitrectomy and of intravenous antibiotics for the treatment of postoperative bacterial endophthalmitis. Endophthalmitis Vitrectomy Study Group. Arch Ophthalmol. 1995;113(12):1479-1496.

25. Chong DY, Anand R, Williams PD, Qureshi JA, Callanan DG. Characterization of sterile intraocular inflammatory responses after intravitreal bevacizumab injection. Retina. 2010;30(9):1432-1440.

26. Sato T, Emi K, Ikeda T, et al. Severe intraocular inflammation after intravitreal injection of bevacizumab. Ophthalmology. 2010;117(3):512-516, 516.e1-e2.

27. Bodnar Z, Clouser S, Mamalis N. Toxic anterior segment syndrome: update on the most common causes. J Cataract Refract Surg. 2012, 38(11):1902-1910.

28. Kahook MY, Kimura AE, Wong LJ, Ammar DA, Maycotte MA, Mandava N. Sustained elevation in intraocular pressure associated with intravitreal bevacizumab injections. Ophthalmic Surg Lasers Imaging. 2009;40(3):293-295.

29. Agrawal S, Joshi M, Christoforidis JB. Vitreous inflammation associated with intravitreal anti-VEGF pharmacotherapy. Mediators Inflamm 2013;2013:943409.

30. Taban M, Singh RP, Chung JY, Lowder CY, Perez VL, Kaiser PK. Sterile endophthalmitis after intravitreal triamcinolone: a possible association with uveitis. Am J Ophthalmol. 2007;144(1):50-54.

31. Casparis H, Wolfensberger TJ, Becker M, et al. Incidence of presumed endophthalmitis after intravitreal injection performed in the operating room: a retrospective multicenter study. Retina. 2014;34(1):12-17.

32. Wickremasinghe SS, Michalova K, Gilhotra J, et al. Acute intraocular inflammation after intravitreous injections of bevakcizumab for treatment of neovascular age-related macular degeneration. Ophthalmology. 2008;115(11):1911-1915.

33. Paul M, Vieillard V, Roumi E, et al. Long-term stability of bevacizumab repackaged in $1 \mathrm{~mL}$ polypropylene syringes for intravitreal administration. Ann Pharm Fr. 2012;7(3):139-154.

34. McCannel CA. Meta-analysis of endophthalmitis after intravitreal injection of anti-vascular endothelial growth factor agents: causative organisms and possible prevention strategies. Retina. 2011;31(4):654-661.

35. Ta CN. Minimizing the risk of endophthalmitis following intravitreous injections. Retina. 2004;24(5):699-705.

36. Lad EM, Moshfeghi DM. Minimizing the risk of endophthalmitis following intravitreal injections. Compr Ophthalmol Update. 2006;7(6):277-284; discussion 285-286.
Clinical Ophthalmology

\section{Publish your work in this journal}

Clinical Ophthalmology is an international, peer-reviewed journal covering all subspecialties within ophthalmology. Key topics include: Optometry; Visual science; Pharmacology and drug therapy in eye diseases; Basic Sciences; Primary and Secondary eye care; Patient Safety and Quality of Care Improvements. This journal is indexed on Submit your manuscript here: http://www.dovepress.com/clinical-ophthalmology-journal

\section{Dovepress}

PubMed Central and CAS, and is the official journal of The Society of Clinical Ophthalmology (SCO). The manuscript management system is completely online and includes a very quick and fair peer-review system, which is all easy to use. Visit http://www.dovepress.com/ testimonials.php to read real quotes from published authors. 